\title{
TREATMENT OF ARTERIAL HYPERTENSION BY PHYSICAL METHODS
}

\author{
Dimo S. Krastev ${ }^{1}$, Ivan Petcov${ }^{1}$, Nikolay Krastev ${ }^{2,4}$, Manol Kalniev², Alexander \\ Apostolov ${ }^{3}$, \\ 1) College of Medicine “J. Filaretova”, Medical University, Sofia, \\ 2) Department of Anatomy and Histology, Medical University, Sofia, \\ 3) Department of Forensic Medicine and Deontology, Medical University, Sofia, \\ 4) Specialized Hospital for Rehabilitation - „Bankya” and „Sapareva Banya”, \\ Sofia, Bulgaria.
}

\section{SUMMARY:}

One of the most important symptoms in many nosological units in the past and present century is the arterial hypertension. It is a widespread symptom leading to very serious consequences, making it an important medical and social issue. In adults arterial hypertension registers within $8-18 \%$, approximately $5 \%$ of cases with arterial hypertension is possible the cause not to be found. In these cases we speak of hypertension of unknown etiology.

Key words: arterial hypertension, cerebrovascular disease, physical treatment

\section{INTRODUCTION:}

In clinical practice it is assumed that values above 140/ $90 \mathrm{~mm} \mathrm{Hg}$ have increased risk of disease, and more than 160/ $95 \mathrm{~mm} \mathrm{Hg}$ talking about arterial hypertension $[1,2,3,4,5$.].

Etiology and pathogenesis of hypertension is very diverse and varied, numerous studies and research papers are devoted to it. It is estimated that $5 \%$ of those with arterial hypertension cannot be determined the reason. Contemporary comprehensions on the occurrence of this symptom are based primarily on two positions, namely interaction of genetic factors and environmental factors.

Physical therapy takes important place mainly in the prevention of hypertension, especially the persons who belong to the group at risk $[3,5,6,7$.$] . Physical therapy is$ applied primarily in the first and second functional stage of the disease.

It aims influencing the many pathogenetic mechanisms leading to the occurrence of the disease $[8,9$.].

\section{Purpose and tasks}

The purpose of this study is, with the help of a different physical factors applied in specialized rehabilitation hospitals "Bankya" and "Sapareva Banya" to monitor therapeutic effect in the treatment of patients with hypertension.
To achieve this goal we set ourselves the following tasks:

1. To influence the pathogenetic mechanisms leading to the occurrence of disease within the patient's experience, and this is achieved by:

a. improve neuro-psychological regulation thru taking the patient out from his home environment and daily routine;

b. restore the imbalance between excitatory and inhibitory cortical processes through various physical factors;

c. improve neuro-vegetative regulation of cardiovascular system.

2. To stimulate the immune system by:

a. Rehabilitation of elasticity of blood vessels wall;

b. training the cardiovascular system;

c. improve metabolism.

\section{MATERIALS AND METHODS}

For this study were used materials from the Specialized Hospital for Rehabilitation - Bankya and "Sapareva Banya". National Health Information Centre" and own observations.

\section{RESULTS:}

The diagnosis of hypertension cannot be placed and based only by measuring the systolic and diastolic blood pressure. It must be supported with evidence from the history, clinical and laboratory studies.

Notable are the different classifications of hypertension. By the suggestion of the WHO, where hypertension occurs in three stages, each of which is divided into two types, $\mathrm{A}$ and $\mathrm{B}$.

I-Grade: A - prehypertensive, B - transient;

II-Grade: A - unstable, B - stable;

III-Grade: A - compensated B - decompensated.

The duration of each state is different, depending on the general condition and associated diseases. In many 
cases, patients may remain for a long time in only one for example the second stage, these are seen mostly in middleaged women.

Hypertension can develop rapidly and lead to severe damage to the kidneys and other organs. Typical are hemorrhage and exudates in the retina, with / or without papilloedema. Postmortem this is characterized with fibernoid arterial necrosis. In such cases it comes to malignant hypertension - a characteristic shape of the course of the disease that progresses rapidly and end is fatal if not recognized and treated promptly

\section{Treatment of hypertension:}

Multiple factors involved in the ethiopathogenesis of hypertension requires therapy to be complex and according to the values of blood pressure and extent of the disease.

\section{Physical therapy}

Physical therapy is most appropriate in the first and second stage of the disease.

We propose the following plan of physical therapy for hypertension:

1. Climate, to it: belong aerotherapy, Sunbathing, (heliotherapy), hydrotherapy and spa;

2. Physiotherapy: it takes major place to prevention and treatment, it refers to the healing gymnastics. To have a better effect is appointed at the earliest stage of the disease.

Complexes building the exercises are with different duration depending on the stage of the disease:

a. 30 to 80 minutes in the first degree hypertension;

b. 20 to 45 minutes in the second degree hypertension;

c. 10 to 20 minutes in the third degree hypertension.

It is important to note that to avoid power exercises connected with exertion and holding the breath. Isometrics do not apply in the third degree of the disease. At this degree preferably are fly gymnastic exercises - performed in full amplitude, with calm and slow pace at full motor muscle relaxation.

Main place in the complex physiotherapy take relaxing - exercises as prevalent - respiratory - exercises.

In this sense, respiratory gymnastics can take a significant place in the healing program - applied individually outside the complex for healing gymnastics. Observe the distraction, when rotating small and large muscle groups of the upper and lower limbs.

It is desirable, gymnastic classes to be group with various appliances.

Throughout the healing gymnastics is necessary to monitor and measure pulse rate and blood pressure, the most appropriate is at the beginning of the complex at peak load and at the end of the complex.

High levels of blood pressures of pulse rate require cutting down the physical activity, and optimization of drug therapy. Information about the heartload gives us the multiplication - of (heart rate by systolic blood pressure), (Dorossiev, D., 1978).

In a complex of medical gymnastics is not desirable to include complex and asymmetric coordination exercises, they lead to overstretch the nervous system, and the exercises associated with sharp movements and bending of the head are - contraindicative.

Recommended sports for people with arterial hypertension depending on the stage are:

- In the first stage: volleyball, tennis, gymnastics, skiing, biking, boating, swimming and skating.

- In the second stage of hypertension: tennis, fishing, mobile games (limited, with less physical and mental stress).

One of the most effective ways to treat patients with hypertension is sanatorium treatment.

\section{DISCUSSION:}

Physical resources are used from antiquity to the present day, as a symptomatic treatment to alleviate the condition of the patient.

Arterial hypertension appears as commonly recognized risk factor for the occurrence of many diseases violating capacity and normal way of life for millions of people in different age groups. Numerous epidemiological studies in recent years have shown a clear connection between arterial hypertension with the occurrence of acute ischemic circulation and cerebral hemorrhage.

Physical factors benefited the basic pathogenic mechanisms lead to the occurrence of hypertension.

They are used to improve cerebral circulation and strengthens condition-reflectoral cortical activity. Physical factors contribute to the elimination of stagnant outbreaks in excitatory vasomotor centers normal dynamics of cortical processes destroy the created pathological cortico-visceral and- viscera-cortical reflexes and condition-reflectoral links and regulates the dynamic balance of the autonomic nervous system. We should not neglect the fact that physical factors favorably affect metabolism (fat and mineral metabolism). Through exercise is normalized and maintaind normal weight of patients which, because of massive obesity lies at the base of hypertension.

By solving these problems physical factors contribute not only to normalize the number of body functions, such as (sleep, appetite, etc..), but also protects the disease. As a result, is achieved significant psychotherapeutic effect, the emotional tone is increased and the confidence of the patient. 


\section{REFERENCES:}

1. Gatev S, Bankov S, Busarov S. factors in the complex treatment "Guide to Physical Therapy" Vol. 2. rehabilitation and some common pub. M\&F Sofia. 1992. [in Bulgarian]

2. Karaneshev G. /editor/ „Medicated gymnastics in some more common diseases" pub. M\&F Sofia. 1975. [in Bulgarian]

3. Kostadinov D. /editor/ „Practical guidance on remedial gymnastics" pub. M\&F Sofia. 1985. [in Bulgarian]

4. Kostadinov D. /editor/ „Physical diseases" pub. M\&F Sofia. 1980. [in Bulgarian]

5. Lang G. „Diseases of the circulatory system" MEDGIZ. M. 1957. [in Russian]

6. Puhlev A, et al. „Blood pressure in the Bulgarian population" pub. BAS Sofia. 1964. [in Bulgarian]

7. Puhlev A, /editor/ „Diseases of the cardiovascular system" pub. $M \& F$ Sofia. 1969. [in Bulgarian]

8. Dorossiev DL. Methodology of physical training, principles of training and exercise prescription. Adv Cardiol. 1978; (24):67-83. [PubMed]

9. Gottheiner V. „Intensive physical training as after-care and prevention of myocardial infarct" [in German] Internist (Berl). 1971 Jun;12(6):236248. [PubMed]

\section{Corresponding author:}

Dimo Stoyanov Krastev, PhD

College of Medicine "Jordanka Filaretova", Medical University-Sofia, Bulgaria. Tel.: +359/2/915 4628 e-mail:dimo_krustev@mail.bg, 